LBL- -32195

DE93 001520

Brief Guide to the MINC-Method

for Modeling Flow and Transport in Fractured Media

\author{
Karsten Pruess \\ Earth Sciences Division \\ Lawrence Berkeley Laboratory \\ University of California \\ Berkeley, California 94720
}

May 1992

This work was supported by the Assistant Secretary for Conservation and Renewable Energy, Geothermal Division, of the U.S. Department of Energy under Contract No. DE-AC03-76SF00098. 


\title{
Brief Guide to the MINC-Method for Modeling Flow and Transport in Fractured Media
}

\author{
Karsten Pruess \\ Earth Sciences Division, Lawrence Berkeley Laboratory \\ University of California, Berkeley, CA 94720
}

\section{What is the MINC-method?}

MINC stands for "Multiple INteracting Continua." It is an approximate method for modeling fluid and heat flow in fractured-porous media, developed by Pruess and Narasimhan $(1982,1985)$ at the Lawrence Berkeley Laboratory. The method is applicable to flow processes in which an important aspect is the exchange of fluid, heat, or chemical species between fractures and unfractured rock. Examples include production and injection operations in fractured geothermal reservoirs, water-flooding, steamflooding, and other methods for enhanced oil recovery from fractured reservoirs, as well as chemical transport and contaminant migration in fractured rock. MINC can only be applied to media in which the fractures are sufficiently well connected so that a continuum treatment of flow in the fracture network can be made.

\section{What are the basic ideas behind "MINC"?}

The method is an extension of the double-porosity concept, originally developed by Barenblatt et al. (1960) and Warren and Root (1963). It is based on the notion that fractures have large permeability and small porosity (when averaged over a reservoir subdomain), while the intact rock (the rock "matrix") has the opposite characteristics. Therefore, any disturbance in reservoir conditions will travel rapidly through the network of interconnected fractures, while invading the matrix blocks only slowly (Pruess and Narasimhan, 1982, 1985; Narasimhan and Pruess, 1988). Based on this observation, the double-porosity concept assumes that "global" flow in a fractured-porous medium occurs exclusively through the fractures, while the matrix blocks may exchange fluid locally with the fractures. This idea is often expressed by postulating a "sugar-cube" model for a fractured-porous reservoir (Figure 1), in which discontinuous matrix blocks are separated from each other by throughgoing fractures. A schematic diagram of the flow geometry in the double-porosity method is given in Figure 2. 


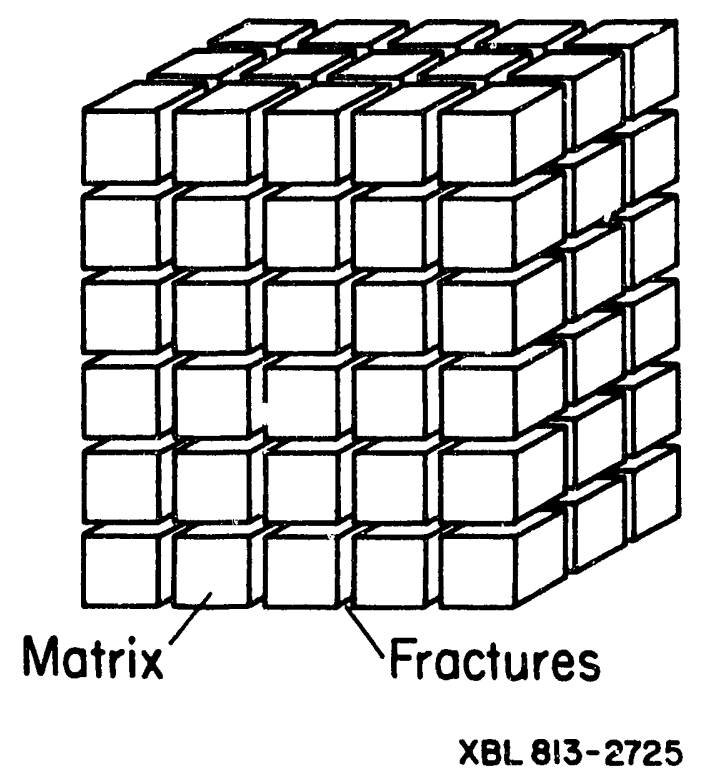

Figure 1. Idealized "sugar cube" model of a fractured porous medium.

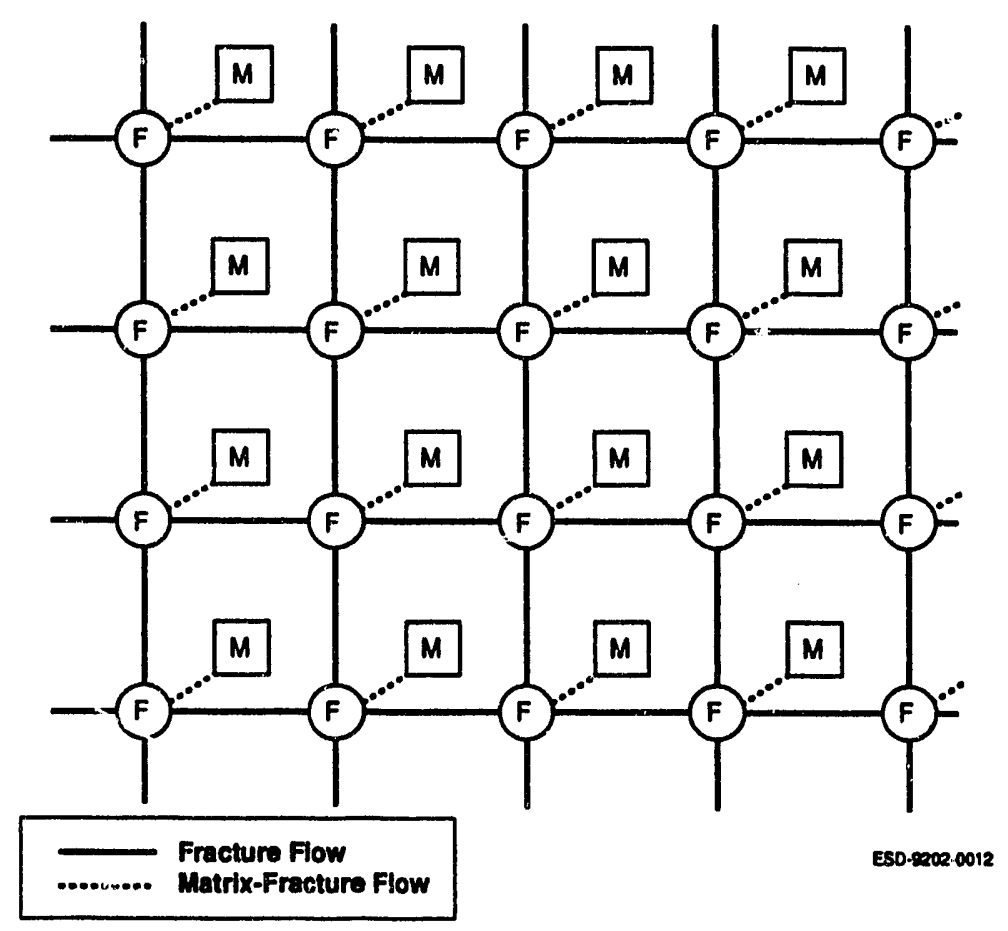

Figure 2. Flow connections in the double-porosity method. 
The crucial point in which the MINC- and the conventional double-porosity methods differ is in the nature of the matrix-fracture exchange (the so-called "interporosity flow"). The double-porosity method assumes that interporosity flow is "quasisteady," i.e., the rate of interporosity flow is proportional to the local difference in average pressures between fractures and matrix, $P_{f}-P_{m}$. This approximation is acceptable for single-phase isothermal flow of fluids with small compressibility, because for these the transient response of matrix blocks will be rapid. The quasi-steady approximation breaks down for non-isothermal and multi-phase flows, where effective diffusivities are small and transient periods of interporosity flow may be very long (decades). The MINCmethod overcomes this limitation by treating interporosity flow in a fully transient way. This is done by computing a numerical approximation to the gradients (of pressure, temperature, etc.) which drive interporosity flow at the matrix-fracture contact.

\section{How does the MINC-method model interporosity flow?}

In order to resolve the gradients driving interporosity flow, it is necessary to employ a suitable discretization of the flow system. On the basis of the different response times of matrix and fractures, this is done as follows. It is assumed that the change in thermodynamic conditions in the matrix blocks is controlled by the distance from the nearest fracture. Therefore, matrix blocks are discretized into a sequence of nested volume elements which are defined on the basis of distance from the fractures, as schematically shown in Figure 3. Continuum \#1 represents the fractures, continuum \#2 represents matrix rock in closest proximity to the fractures, continuum \#3 represents matrix rock at larger distance, etc. In response to an imposed disturbance in the fracture system (e.g. a pressure drop from opening of wells, a change of phase composition and/or temperature from injection of water), fluid and/or heat can migrate in the matrix blocks outward towards the fractures, or inward away from the fractures. Thus, interporosity flow is approximated as being one-dimensional. When only two continua are defined it is easy to show that the MINC-method reduces to the conventional double-porosity approach.

The concept of partitioning the rock matrix into sub-continua based on the distance from the fractures is not limited to regularly-shaped matrix blocks. It can be just as easily applied to irregular fracture distributions (see Figure 4).

\section{How is the MINC-method applied to flow problems?}

For flow modeling the above mentioned discretization scheme must be applied in a quantitative way. This is most easily accomplished with an integral finite difference method, in which space discretization is made directly from integral statements of mass 


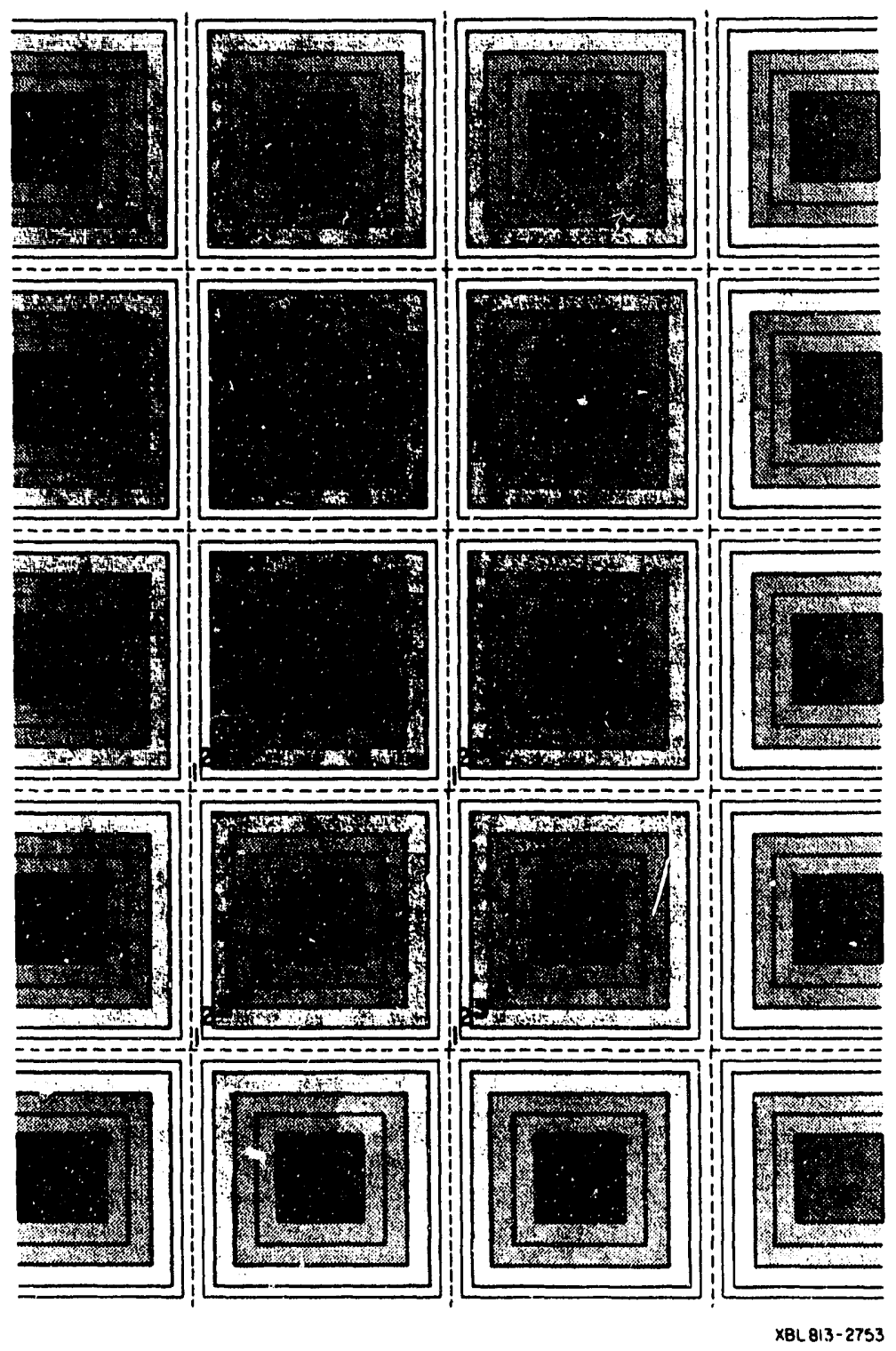

Figure 3. MINC-discretization for a fractured norous medium.

and energy balance equations rather than going through partial differential equations (Narasimhan and Witherspoon, 1977). With integral finite differences, the spacediscretized geometry of a flow problem is specified by means of a list of grid block volumes, interface areas, and nodal distances. There is no need to make reference to a global coordinate system. This coordinate-free approach provides a great deal of flexibility. Discretizations for arbitrary irregular flow geometry, heterogeneous and fractured media, and higher-order differencing schemes can all be implemented through appropriate specification of geometry data without any coding changes in the simulation program. 


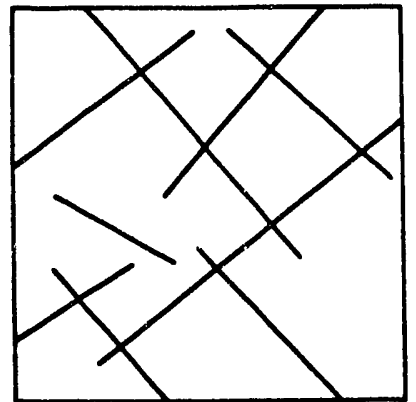

a. Fractures

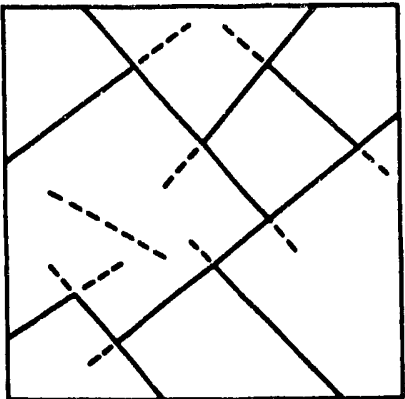

b.Connected fractures

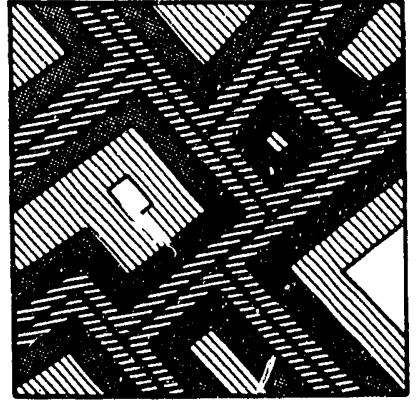

c.MINC partitioning

xaL $2211-2610$

Figure 4. MINC concept for an arbitrary irregular fracture distribution.

The first step in implementing the MINC method is to develop a calculational mesh just as would be done for an unfractured porous medium. This "primary" mesh is then processed to generate a "secondary" mesh which includes the additional volume element and flow interface data needed to describe interporosity flow. The pre-processing is done with a computer program "GMINC" (Pruess, 1983), which requires data on fracture distribution (equivalently: sizes and shapes of matrix blocks). Subsequently the mesh data is merged into an input file for integral-finite difference simulators such as TOUGH/TOUGH2 (Pruess, 1987, 1991), STMFLD1 (Pruess, 1988), or STMVOC (Falta and Pruess, 1991; Falta et al., 1992a,b), and the flow simulation proceeds in the same way as it would for a porous flow problem. Thus the "mechanics" of implementing and running the MINC-method are very simple. The difficult part is in the judgement as to whether the MINC-method will in fact provide a reasonable approximation to the flow problem at hand. The two crucial questions to ask in this context are: (1) Is it (approximately) true that global flow in the reservoir occurs only through the fractures, not the matrix blocks? (2) Is it (approximately) true that (changes in) thermodynamic conditions in the matrix blocks will depend only on the distance from the nearest fracture?

An explicit discretization of all matrix blocks, as depicted in the idealized geometry shown in Figure 3, would still generate prohibitively large numbers of gric blocks in most reservoir flow problems. In practical applications of the MINC method, space discretization is simplified further by lumping all "like" continua within a reservoir subdomain into a single computational grid block. For example, if a grid block of the primary mesh contains many matrix blocks, all matrix regions labeled " 2 " in Figure 3 are lumped into one calculational olock of the secondary mesh, all regions labeled " 3 " are lumped into another block, etc. It is this aggregation of flow regions, subject to the above 
caveats, which achieves a sufficient reduction in the number of calculational grid blocks to make the MINC approximation applicable to practical reservoir problems.

There is an important class of multiphase flow problems in which fluid phases are to a large extent segregated between fractures and matrix blocks, so that the geometry for global flow may be different for different phases. An important example is the flow of water and air in fractured unsaturated zones, where capillary forces dictate that the wetting phase (water) flows primarily through matrix blocks, while the non-wetting phase (air) flows primarily through the fractures (Wang and Narasimhan, 1985). Similar flow structures may occur in vapor-dominated geothermal reservoirs (Pruess, 1985) and in waterflooding of fractured petroleum reservoirs (Firoozabadi and Hauge, 1990; Horle et al., 1990). Systems with different global flow geometry for different phases are termed "dual permeability." The MINC method can be generalized to include global matrixmatrix flow connections in addition to fracture-fracture connections, so that it can be applied to dual-permeability systems as well (Wang and Narasimhan, 1985, 1990; Pruess, 1985). This leads to a flow geometry as shown in Figure 5. We emphasize that great care must be exercised in the specification of flow geometry for dual-permeability systems to avoid unphysical behavior (Pruess, 1990). An example of a dual-permeability mesh employing MINC for the fractured regions is given in Figure 6 (from Wang and Narasimhan, 1990).

\section{Some methodological variations}

The basic premise of the MINC method - that thermodynamic conditions in matrix blocks are controlled by the distance from the nearest fracture - does not necessarily require a space-discretized numerical approach. The same concept has been utilized in a number of analytical or semi-analytical treatments of flow in fractured media. Lai et al. (1983) developed an analytical formulation based on Laplace transforms for well test analysis in single-phase fractured reservoirs. Pruess and Wu (1989) presented a semianalytical method for simulating fluid and heat flow in fractured reservoirs, in which an approximate analytical treatment of transient diffusion in matrix blocks was embedded in a finite-difference representation of global flow in the fractures. Zimmerman and Bodvarsson (1989) used an integral method for an approximate treatment of diffusion in matrix blocks. This provided a source term in the equation for flow in the fracture system which allowed a description of transient fracture-matrix exchange without sub-gridding of matrix blocks (Zimmerman, Chen and Bodvarsson, 1992).

A fully numerical treatment of all fracture and matrix flow through finite difference space discretization, as was originally proposed in the MINC concept, offers the greatest 


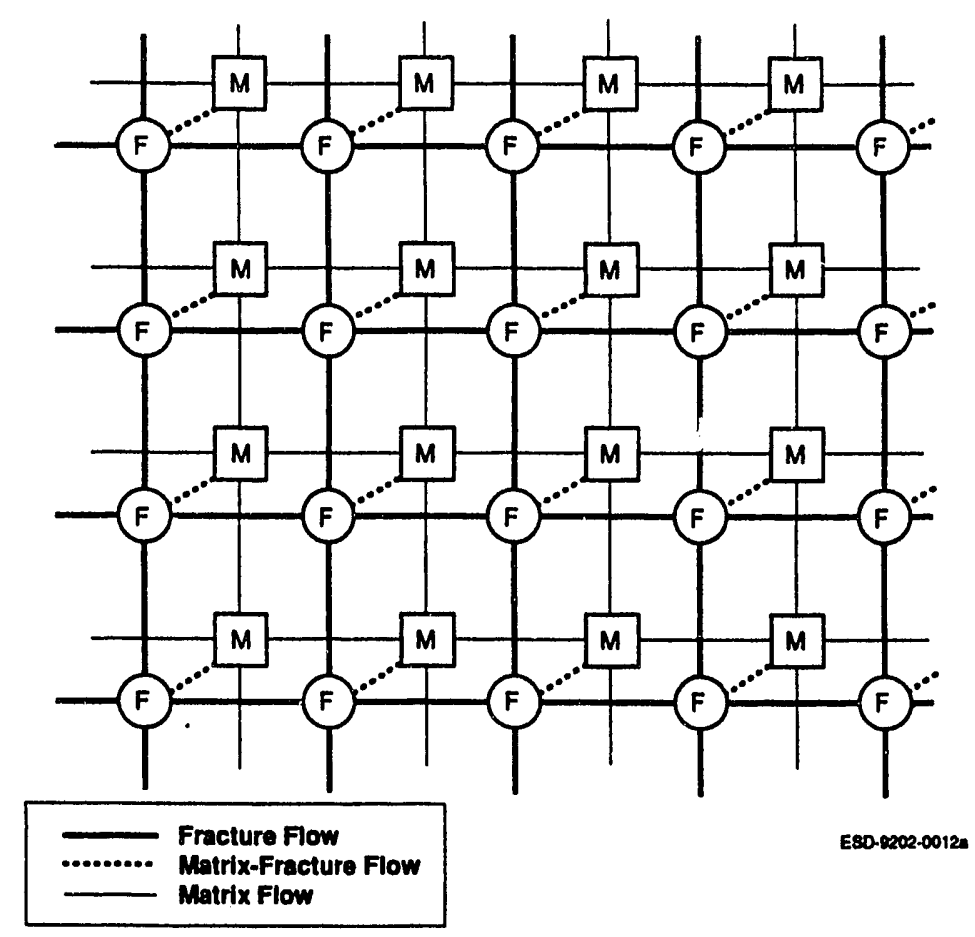

Figure 5. Flow connections for a "dual permeability" system.

flexibility and is applicable to highly nonlinear flow problems. The price to be paid for this flexibility is a considerable increase in the number of grid blocks and computation time, typically by a factor of five, relative to porous medium flow problems. The analytical and semi-analytical approximations to interporosity flow obviate the need for matrix block subgridding, and can model flow in fractured media with little additional computational work. However, these methods are more limited in scope and can be applied only to simpler subclasses of flow problems.

\section{What applications of the MINC-method have been made?}

Most of the applications of the MINC method to date have been directed towards the study of processes and recovery mechanisms in geothermal reservoirs. Fundamental studies of thermodynamic conditions and physical processes in vapor-dominated geothermal reservoirs were presented by Pruess and Narasimhan (1982, 1985), Pruess (1983, 1985), Pruess, Celati, Calore and D'Amore (1985), Calore, Pruess and Celati (1986), Bodvarsson and Gaulke (1987) and Pruess, Celati, Calore and Cappetti (1987). Heat 


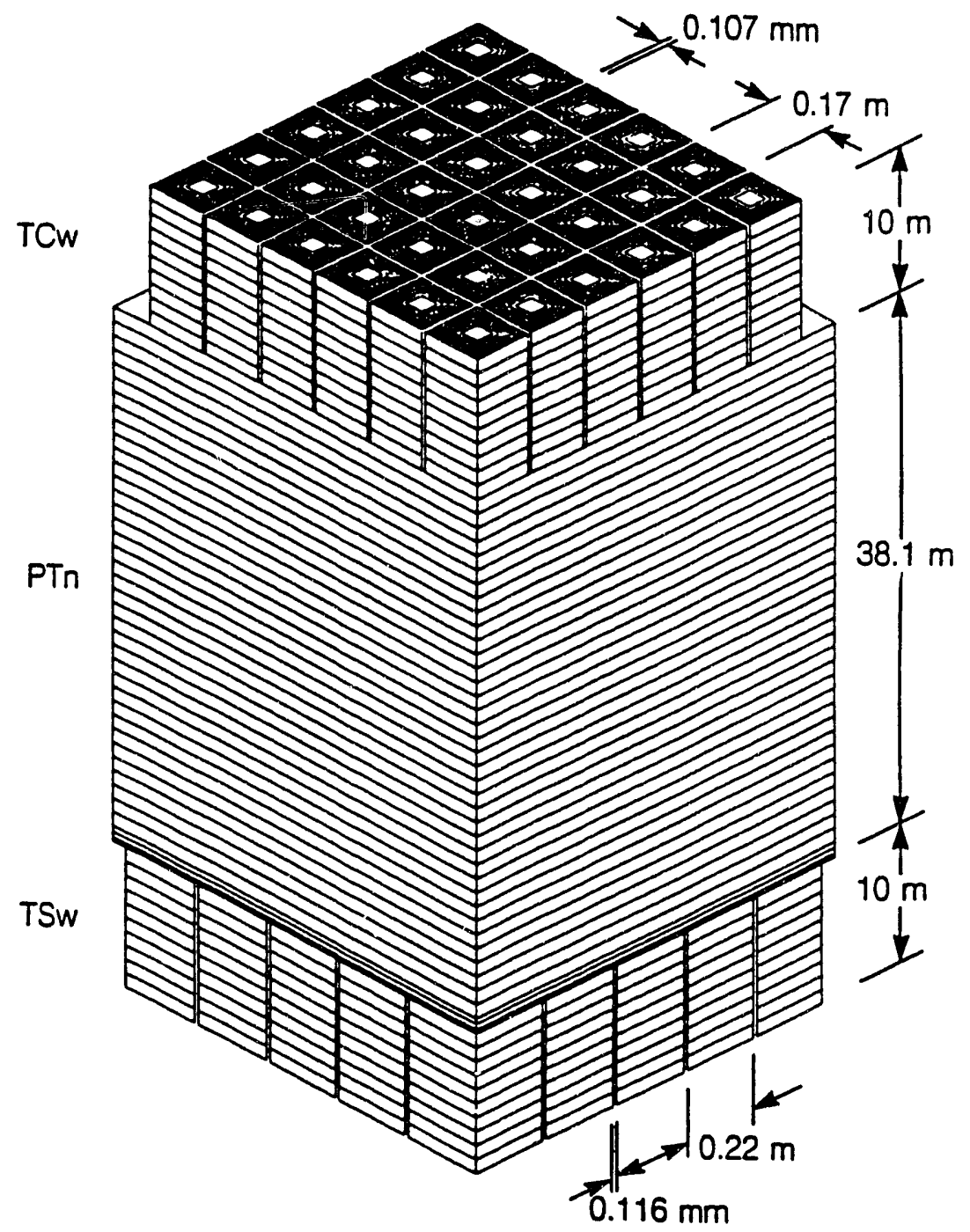

X8L 865-1793

Figure 6. Discretization of a layered system consisting of alternating fractured-porous and unfractured units (from Wang and Narasimhan, 1990).

extraction and reinjection in single-phase liquid geothermal reservoirs was studied by Bodvarsson, Pruess and O'Sullivan (1985). Laboratory heat extraction experiments were modeled by Lam, Hunsbedt, Kruger and Pruess (1988). Bodvarsson, Gaulke and Ripperda (1989) presented the development of a MINC-based reservoir model for The Geysers vapor-dominated geothermal field. Ishido et al. (1992) used a MINC approach for analyzing pressure transient data from the Sumikawa geothermal field.

Some applications of MINC have been made in areas other than geothermal reservoir engineering. Waterflooding of fractured hydrocarbc $\eta$ reservoirs was modeled by $\mathrm{Wu}$ 
and Pruess (1988) and by Beckner et al. (1991). In unpublished work this author applied MINC to simulate enhanced oil recovery by steamflooding from a naturally fractured reservoir. An application to chemical transport in fractured rock was presented by Neretnieks and Rasmuson (1984). Wang and Narasimhan (1990) used a MINC discretization to study water infiltration in thick unsaturated zones consisting of alternating porous and fractured-porous layers (see Figure 6).

A number of studies have examined issues relating to the accuracy of the MINC approximation. These include papers by Lai et al. (1986), Pruess and Wu (1989), Pritchett and Garg (1990) and Chen et al. (1991).

\section{Conclusion}

MINC is a powerful and flexible method for modeling of complex flow processes in fractured porous media. Where applicable it can yield large savings in computer time in comparison to approaches with more detailed space discretization. MINC is not a patent recipe, however. It is an approximation whose applicability must be carefully evaluated for any given flow problem.

A capability for MINC simulations is available through a number of public-domain codes, and through commercial software vendors (e.g., S-Cubed, LaJolla, California; Computer Modelling Group, Calgary, Alberta).

\section{Acknowledgement}

Thanks are due to C. H. Lai and M. Shook (INEL) for a careful review of the manuscript. This work was supported by the Assistant Secretary for Conservation and Renewable Energy, Geothermal Division, of the U.S. Department of Energy under contract DE-AC03-76SF00098.

\section{References}

Barenblatt, G. E., I. P. Zheltov and I. N. Kochina, (1960). Basic Concepts in the Theory of Seepage of Homogeneous Liquids in Fractured Rocks, J. Appl. Math., (USSR), 24, (5) 1286-1303.

Beckner, B. L., H. M. Chan, A. E. McDonald, S. O. Wooten and T. A. Jones, (1991). Simulating Naturally Fractured Reservoirs Using a Subdomain Method, paper SPE-21241, presented at the Eleventh SPE Symposium on Reservoir Simulation, Anaheim, CA., February.

Bodvarsson, G. S., K. Pruess and M. J. O'Sullivan, (1985). Injection and Energy Recovery in Fractured Geothermal Reservoirs, Society of Petroleum Engineers Journal, 25, (2) 303-312, April. 
Bodvarsson, G. S. and S. Gaulke, (1987). Effects of Noncondensible Gases on Fluid Recovery in Fractured Geothermal Reservoirs, SPE Reservoir Engineering, 335342, August.

Bodvarsson, G. S., S. Gaulke and M. Ripperda, (1989). Some Considerations on Resource Evaluation at The Geysers, Geothermal Resources Council, Transactions, 13, 367-375.

Calore, C., K. Pruess and R. Celati, (1986). Modeling Studies of Cold Water Injection into Fluid-Depleted, Vapor-Dominated Geothermal Reservoirs, paper presented at Eleventh Workshop Gecthermal Reservoir Engineering, Stanford University, Stanford, Ca., January.

Chen, J., M. A. Miller and K. Sepehrnoori, (1991). Comparisons of Counter-Current Imbibition Transfer Functions in Dual-Porosity Models of Naturally Fractured Reservoirs, In Situ, 15, (2), 115-147.

Falta, R. W. and K. Pruess, (1991). STMVOC User's Guide, Lawrence Berkeley Laboratory Report LBL-30758, June.

Falta, R. W., K. Pruess, I. Javandel and P. A. Witherspoon, (1992). Numerical Modeling of Steam Injection for the Removal of Nonaqueous Phase Liquids from the Subsurface. 1. Numerical Formulation, Water Resources Research, 28, (2) 433-449.

Falta, R. W., K. Pruess, I. Javandel and P. A. Witherspoon, (1992). Numerical Modeling of Steam Injection for the Removal of Nonaqueous Phase Liquids from the Subsurface. 2. Code Validation and Application, Water Resources Research, 28, (2) 451 465.

Firoozabadi, A. and J. Hauge, (1990). Capillary Pressure in Fractured Porous Media, Journal of Petroleum Technology, 784-791, June.

Horle, T., A. Firoozabadi and K. Ishimoto, (1990). Laboratory Studies of Capillary Interactions in Fracture/Matrix Systems, SPE Reservoir Enginee-ing, 353-360, August.

Ishido, T., T. Kikuchi, Y. Yano, Y. Miyazaki, S. Nakao and K. Hatakeyama, (1992). Analysis of Pressure Transient Data from the Sumikawa Geothermal Field, Proceedings, Seventeenth Workshop Geothermal Reservoir Engineering, Stanford University, Stanford, CA, January.

Lai, C. H., G. S. Bodvarsson, C. F. Tsang and P. A. Witherspoon, (1983). A New Model for Well Test Data Analysis for Naturally Fractured Reservoirs, paper SPE-11688, presented at the 1983 California Regional Meeting of the SPE, Ventura, Ca.

Lai, C. H., K. Pruess and G. S. Bodvarsson, (1986). On the Accuracy of the MINC Approximation, Lawrence Berkeley Laboratory Report LBL-21025, Berkeley, Ca., February.

Lam, S. T., A. Hunsbedt, P. Kruger and K. Pruess, (1988). Analysis of the Stanford Geothermal Reservoir Model Experiments Using the LBL Reservoir Simulator, Geothermics, 17, (4) 595-605.

Narasimhan, T. N. and P. A. Witherspoon, (1977). Numerical Model for SaturatedUnsaturated Flow in Deformable Porous Media. 1. Theory, Water Resources Research, 13, (3) 657-664, June. 
Narasimhan, T. N. and K. Pruess, (1988). MINC: An Approach for Analyzing Transport in Strongly Heterogeneous Systems, Groundwater Flow and Quality Modeling, E. Custodio, A. Gurgui and J. P. Lobo Ferreira (eds.), D. Reidel Publishing Company, Dordrecht, 375-391.

Neretnieks, I. and A. Rasmuson, (1984). An Approach to Modeling Radionuclide Migration in a Medium with Strongly Varying Velocity and Block Sizes along the Flow Path, Water Resources Research, 20, (12) 1823-1836, December.

Pritchett, J. W. and S. K. Garg, (1990). On Similitude, Heat Conduction and Two-Phase Flow in Fractured Porous Media, paper presented at Fifteenth Workshop on Geothermal Reservoir Engineering, Stanford University, Stanford, Ca., January.

Pruess, K., (1983). GMINC - A Mesh Generator for Flow Simulations in Fractured Reservoirs, Lawrence Berkeley Laboratory Report LBL-15227, Berkeley, Ca., March.

Pruess, K., (1983). Heat Transfer in Fractured Geothermal Reservoirs with Boiling, Water Resources Research, 19, (1) 201-208, February.

Pruess, K., (1985). A Quantitative Model of Vapor Dominated Geothermal Reservoirs as Heat Pipes in Fractured Porous Rock, Geothermal Resources Council, Transactions, 9, part II, 353-361, August.

Pruess, K., (1987). TOUGH User's Guide, Nuclear Regulatory Commission, Report NUREG/CR-4645, June 1987 (also Lawrence Berkeley Laboratory Report LBL20700, Berkeley, Ca., June).

Pruess, K., (1988). STMFLD1 User's Guide, Report, Flow Science Inc., Pasadena, Ca.

Pruess, K., (1990). Numerical Modeling of Gas Migration at a Proposed Repository for Low and Intermediate Level Nuclear Wastes at Oberbauenstock, Switzerland, Lawrence Berkeley Laboratory Report LBL-25413, NDC-5.

Pruess, K., (1991). TOUGH2 - A General-Purpose Numerical Simulator for Multiphase Fluid and Heat Flow, Lawrence Berkeley Laboratory Report LBL-29400, May.

Pruess, K. and T. N. Narasimhan, (1982). On Fluid Reserves and the Production of Superbeated Steam from Fractured, Vapor-Dominated Geothermal Reservoirs, Journal Geophysical Research, 87, (B11) 9329-9339.

Pruess, K., R. Celati, C. Calore and F. D'Amore, (1985). $\mathrm{CO}_{2}$ Trends in the Depletion of the Larderello Vapor-Dominated Reservoir, Lawrence Berkeley Laboratory Report LBL-19092, presented at Tenth Workshop Geothermal Reservoir Engineering, Stanford University, Stanford, Ca.

Pruess, K. and T. N. Narasimhan, (1985). A Practical Method for Modeling Fluid and Heat Flow in Fractured Porous Media, Society of Petroleum Engineers Journal, 25, (1) 14-26, February.

Pruess, K., R. Celati, C. Calore and G. Cappetti, (1987). On Fluid and Heat Flow in Deep Zones of Vapor-Dominated Geothermal Reservoirs, paper presented it Twelfth Annual Workshop Geothermal Reservoir Engineering, Stanford University, Stanford, Ca., January (also Lawrence Berkeley Laboratory Report LBL-22810). 
Pruess, K. and Y. S. Wu, (1989) A New Semianalytical Method for Numerical Simulation of Fluid and Heat Flow in Fractured Reservoirs, paper SPE-18426, presented at Tenth SPE Symposium on Reservoir Simulation, Houston, TX; to appear in SPE Reservoir Engineering, 1991.

Wang, J. S. Y. and T. N. Narasimhan, (1985). Hydrologic Mechanisms Governing Fluid Flow in a Partially Saturated, Fractured, Porous Medium, Water Resources Research, 21, (12) 1861-1874, December.

Wang, J. S. Y. and T. N. Narasimhan, (1990). Fluid Flow in Partially Saturated, Welded-Nonwelded Tuff Units, Geoderma, 46, 155-168.

Warren, J. E. and P. J. Root, (1963). The Behavior of Naturally Fractured Reservoirs, Society Petroleum Engineers Journal, 245-255, September; Transactions, AIME, 228.

Wu, Y. S. and K. Pruess, (1988). A Multiple-Porosity Method for Simulation of Naturally Fractured Petroleum Reservoirs, SPE Reservoir Engineering, 3, 327-336, February.

Zimmerman, R. W. and G. S. Bodvarsson, (1989). Integral Method Solution for Diffusion into a Spherical Block, Journal of Hydrology, 111, 213-224.

Zimmerman, R. W., G. Chen and G. S. Bodvarsson, (1992). A Dual-Porosity Reservoir Model with an Improved Coupling Term, Lawrence Berkeley Laboratory Report LBL-31693, presented at Seventeenth Workshop on Geothermal Reservoir Engineering, Stanford University, Stanford, Ca., January. 

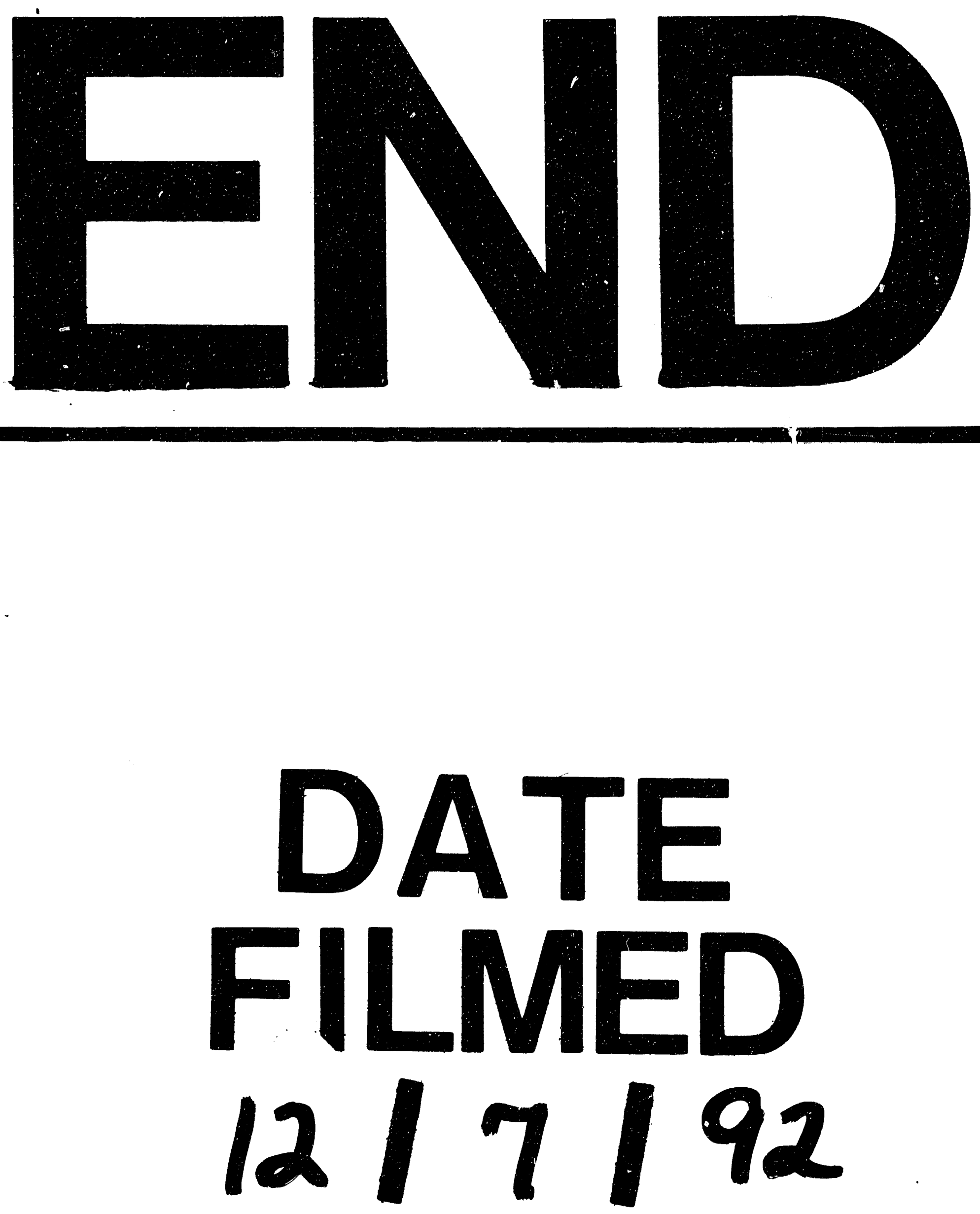
